\title{
La educomunicación en la universidad virtual: retos del modelo
}

\author{
Nelly TOVAR TORRES \\ Universidad Nacional Abierta y a Distancia (Colombia) \\ nelly.tovar@unad.edu.co
}

Recibido: $22 / 11 / 2012$

Aceptado: 23/01/2013

\begin{abstract}
Resumen
La universidad virtual se erige desde el discurso posmodernista en global, innovadora e integradora, sin modificar las viejas estructuras de la educación presencial. Esta circunstancia y la cada vez transformación de la educación como una empresa y no como un derecho, tiene efectos evidentes en la cultura y la sociedad, lo que será analizado críticamente a través de la educomunicación. Así, este artículo presenta los avances de la investigación desde las categorías de generación de conocimiento e interacción entre docente-estudiante-técnico, y cómo éstas le plantean nuevos retos al modelo virtual.

Palabras clave: Educomunicación, Universidad Virtual, Docencia Virtual, Nuevo Conocimiento y Enseñanza/Aprendizaje.
\end{abstract}

\section{Educommunication in the Virtual University: Challenges of the model}

\begin{abstract}
Virtual university raises, from the post modern discourse, global, innovating, and integrating without modifying the old in attendance educational structures. This circumstance and the every other time education transformation like an enterprise and not as a right has its evident effects in culture and society which will be critically analyzed through educommunication. Thus, this paper presents the progress of the investigation from the categories of knowledge generation and the teacher - student - technical interact and how they will present new challenges to the virtual model.
\end{abstract}

Keywords: Educommunication, Virtual University, Virtual Teaching, New Knowledge and Teaching/Learning.

\section{Referencia normalizada}

TOVAR TORRES, Nelly (2013): "La educomunicación en la universidad virtual: retos del modelo". Estudios sobre el Mensaje Periodístico. Vol. 19, Núm. especial marzo, págs.: 491-499. Madrid, Servicio de Publicaciones de la Universidad Complutense

Sumario: 1. Introducción. 2. Metodología. 3. Generación de conocimiento. 4. Interacción docente-estudiante-técnico. 5. Retos de la modalidad virtual. 6. Conclusiones. 7. Referencias bibliográficas

\section{Introducción}

El análisis y la reflexión de esta fase documental de la investigación, a partir de las categorías establecidas, parte de los aportes que Prieto hace a la comunicación educativa, comunicación en la educación o educomunicación, al relacionarla con procesos de socialización democrática, reconocimiento a la diversidad cultural y centrada en la promoción y acompañamiento del aprendizaje o "mediación dialógica". Esta relación, que es esencialmente pedagógica se da entre seres que se comunican, que interactúan, que se construyen en la interlocución. "No se puede hacer educación sin comunicación, lo que significa pensar a ésta en clave de aprendizaje". (Prieto, 2006: 86)

Partiendo de reconocer la importancia de la comunicación en los procesos educativos, la investigación iniciada en el año 2011, constituye un esfuerzo por contribuir 
al mejoramiento de la calidad de la educación superior en el país, a partir de la pregunta ¿Qué papel desempeña la universidad virtual en la sociedad a partir de elementos educomunicativos como la generación de conocimiento, los medios/ tecnologías y la interacción docente-estudiante-técnico?

El artículo presenta la primera fase del estudio, que consiste en la revisión documental desde las categorías definidas inicialmente en la pregunta investigativa: generación de conocimiento, medios/tecnologías, interacción docente-estudiante-técnico. En el proceso investigativo surgieron categorías emergentes como la institucionalidad y los contextos local/nacional, que desarrollaron en documentos ampliados.

Los resultados de la primera fase investigativa, permitieron establecer una serie de características propias de la aplicación del modelo, denominadas: Retos de la modalidad virtual, referidas a los aspectos identificados como débiles en este tipo de educación, constituyéndose en variables, que luego serán profundizadas en los grupos de discusión.

\section{Metodología}

En el desarrollo de la investigación, se partió de la observación y reflexión sistemática sobre la realidad de la educación superior y específicamente de la universidad virtual en la sociedad, con una herramienta analítica denominada educomunicación, la que permitió construir una matriz de análisis con los criterios de inclusión de las fuentes de información: libros e investigaciones relacionadas con el uso de las TIC en la educación, comunicación y educación, nuevas narrativas y otras temáticas pertinentes; artículos de investigación publicados entre los años 2005 y 2012 de las bases de datos Erin y Fuente académica; documentos de universidades con modalidad virtual, como proyectos institucionales y curriculares, syllabus, estados del arte de la investigación, estándares e informes técnicos; plataforma Moodle; cursos en diversas plataformas educativas, y otros que condujeron a una primera conceptualización de la temática general.

Tipo de Investigación: De acuerdo a las diversas clasificaciones, la presente investigación está ubicada según la naturaleza y tratamiento de los datos como predominantemente cualitativa y de tipo inductiva. Al decir que su énfasis es cualitativo, se refiere a que se orienta al estudio de las acciones humanas y de la vida social utilizando la metodología interpretativa. Además es considerada de tipo mixta, porque utiliza la investigación documental y la investigación de campo. En síntesis, es una investigación con "un método de pensamiento crítico" (Tamayo, 2003: 38) que permite descubrir datos, sucesos, relaciones o leyes en un campo del conocimiento.

Recolección de datos - Técnicas e instrumentos: Los pasos de la etapa de recolección de datos, implicaron: elaboración de la matriz con criterios definidos para la elección de las fuentes, recolección de la información de manera sistemática, análisis deductivo bajo categorías establecidas, ordenamiento conceptual y teorización.

Teniendo en cuenta la necesidad de articular lo epistemológico con los métodos de indagación se plantearon las siguientes técnicas e instrumentos de investigación: revisión documental y grupos de discusión. 
Las fuentes documentales se definieron de acuerdo con la pregunta y los objetivos de la investigación. Igualmente, la matriz con las categorías analíticas y luego la emergencia de variables nominales-empíricas y preguntas orientadoras, facilitaron la sistematización de los datos.

En los grupos de discusión, siguiente etapa del proceso investigativo, se trabajará con la comunidad académica. Se trata de llevar a -un grupo de directivos, docentes, estudiantes y egresados - a conversar en una situación de laboratorio - espacio cerrado al exterior. Esta técnica, es una simulación de un pedazo de realidad en la que las relaciones entre los sujetos de la sociedad se da a través de la comunicación, y ésta, a su vez, se puede encontrar en la conversación. En los contenidos del discurso grupal se reproducen las representaciones de la realidad y la información en forma de discursos individuales que chocan y se escuchan, y a su vez, son usados por los mismos participantes en forma cruzada, contrastada y enfrentada. (Galindo, 1998: 75-85)

Según lo anterior y siendo coherentes con el campo del saber de la comunicación, la propuesta metodológica investigativa retoma y desarrolla técnicas propias de la investigación social, de la cultura y la comunicación, en un intento por hacer evidente la necesidad de temáticas, métodos y objetivos de investigación que aporten a un conocimiento propio y socialmente significativo.

\section{Generación de conocimiento}

La universidad virtual entra al reino de lo inmaterial, de lo atemporal, al dominio del simulacro y la simulación que como parte del presente inmediato, se ha convertido en la metáfora por excelencia de la denominada posmodernidad, si es que algún día hubo modernidad para los países indoamericanos. Gran parte de las propuestas de universidad virtual al plantearse como innovadoras, diferenciadas y diferenciadoras aplican políticas de cambio desde la forma y la formalidad, "alimentando más la fantasía del saber que el saber mismo" (Lora, et al, 2003: 70) y adoptando de manera acrítica conceptos y modelos poco pertinentes en aras de la productividad y competitividad; un claro ejemplo, es el afán de muchas entidades de educación superior por obtener certificaciones y acreditaciones de alta calidad, haciendo evidente que quieren ser medidas como lo hacen hoy las empresas que necesitan competir en el mercado.

Un factor importante que en las últimas décadas, incide en la generación de conocimiento, es el relacionado con el "descentramiento y deslocalización de los saberes", (Barbero, s.f: web) pues los discursos liberados de las instituciones y de las figuras de poder tradicionales, circulan hoy por las redes de manera dispersa y fragmentada.

Es así, que el discurso pensado, hablado y escrito, que se constituyó en el eje central de la evolución humana, va perdiendo su estatus y queda cada vez más subordinado a la imagen. La cultura centrada en torno al libro, existe ahora al margen de la gran cantidad de información disponible en internet, en los medios de comunicación, en las bibliotecas electrónicas, en el software y videojuegos educativos, en los mundos virtuales, y demás formatos electrónicos. Y es que el paso de la textualidad a la hipertextualidad, presente en las comunicaciones electrónicas, ha modificado los procesos de lectura-escritura; la idea debe ser clara, concisa, sintética, no es posible el despliegue reflexivo y menos el cuidado en la escritura, no hay mucho tiempo para la 
corrección, y son válidos los saltos temáticos en lugar del orden consecutivo y lineal característico del texto, difundiéndose el uso de abreviaciones y símbolos.

Lo anterior sin embargo, cuestiona no sólo las formas cómo se produce en estos tiempos el conocimiento, sino y especialmente, qué tan pertinente resulta. No parece, que la liberación de gran cantidad de información y datos, antes inaccesibles al público en general sea suficiente para propiciar cambios cualitativos en el conocimiento. ¿Qué le plantea la universidad virtual a la sociedad al respecto?

La generación de nuevo conocimiento que es inherente al proceso formativo, debería estar presente también en el aula, así sea virtual. Cuando no se produce conocimiento, la educación se centra en el viejo esquema de lo transmisivo, desvirtuando el carácter de superior o universitario. De igual modo, el modelo virtual no ha logrado incorporar en sus procesos pedagógicos las transformaciones dadas en la sociedad, especialmente en los jóvenes, frente a la elaboración y apropiación de los saberes presentes en los nuevos medios, y en los cuales se pone en juego como plantea Deleuze, un pensamiento rizomático.

La educación superior y con mayor razón los modelos virtuales no pueden seguir basándose en la transmisión, la educación debe enfocar sus estrategias en la transacción. La clave para interpretar los cambios es "enseñar a aprender", es decir el docente se convierte en un mediador entre la inmensa cantidad de información disponible en la actualidad y sus estudiantes. (Piscitelli, 2011: web)

\section{Interacción docente-estudiante-técnico}

El cambio de la presencialidad a la virtualidad implica a su vez, una transformación radical en el rol del maestro, sobre el que recae la principal responsabilidad académica, solo que ahora con un nuevo actor/rol, el técnico. En este proceso de cambio de perfiles, el rol del técnico se aleja cada vez más del pedagógico y el del docente se acerca cada vez más al del técnico, ya que finalmente este último debe resolver las contingencias propias de los sistemas informáticos (abre-cierra foros y evaluaciones, cambia fechas de acuerdo a las agendas, verifica que la programación sea la adecuada, reabre actividades cuando hay problemas de corte de energía o inestabilidad de la plataforma), dejando de lado los procesos académicos.

Esta relación docente-estudiante-técnico se vuelve compleja, puesto que la universidad virtual, preocupada más por mantener el sistema de conexión, columna vertebral del modelo, deja de lado lo esencial para resolver lo urgente. En este orden, lo técnico provee los recursos necesarios para evitar el colapso.

Se debe hacer un análisis más detallado, acerca de situaciones como la no presencia física y luego la ausencia del docente en los procesos de enseñanza-aprendizaje. Frente a lo primero; se pierden los procesos de información extralinguística: la mirada, los gestos espontáneos, el lenguaje corporal, el timbre y la entonación de la voz, que representan un porcentaje importante de la comunicación humana, y que son elementos trascendentales a la hora tanto del malentendido como de la desinhibición. Esa información perdida por el medio, esa riqueza que brinda la presencia, esa amplitud de tonos, matices, timbres, entonaciones, es la que aún no está presente en la educación virtual y que vuelve la experiencia de la interpretación una experiencia difícil y proclive al error. 
Luego, la ausencia del docente, relativa a la imposibilidad de atender simultáneamente a cientos de estudiantes en uno o varias cursos, situación que desborda las posibilidades físicas y mentales del mejor intencionado. A cambio se ponen a disposición algunos recursos didácticos, especialmente elementos técnicos asincrónicos que no logran generar procesos de interacción suficientes y oportunos, ocasionando abandono institucional hacia el estudiante, al punto de rayar con el autodidactismo, pues no se valora lo suficiente la mediación dialógica con el docente, al considerar en la práctica, que el estudiante puede prescindir de él. Un interés verdadero en sentido contrario, implicaría reconocer y facilitar las condiciones a los docentes para apoyar permanentemente los procesos de enseñanza-aprendizaje, que es realmente lo que solicitan los alumnos en las diversas actividades académicas.

\section{Retos de la modalidad virtual}

Masificada: el acto de individualizar o masificar en la educación, se refiere a esos presupuestos equivocados heredados de la modernidad que consideran que todos los estudiantes son iguales; por tanto tienen las mismas capacidades, las mismas necesidades, aprenden al mismo ritmo y de la misma manera. Este desconocimiento de los talentos naturales, de los gustos y potencialidades personales le ha hecho mucho daño a la sociedad en general, porque no se fortalece la creación y recreación del conocimiento. No es un problema de aprender o no, es que cada persona está dispuesta a aprender algunas cosas más que otras (Durán, 2005: web). La masificación lleva a la uniformización del saber, entendido éste último como algo relacionado con la memoria, con las competencias o con la recursividad. Y aunque resulta más fácil educar en masa que individualmente, los resultados finales son de un fracaso rotundo (Ospina, 2012: 76).

Estandarizada: Es un procedimiento utilizado para corroborar el apropiado funcionamiento de maquinarias, equipos, productos y empresas de acuerdo a unos parámetros establecidos. Sin embargo con la globalización, se viene aplicando al conocimiento y a las diversas formas de pensamiento, por lo que el término está muy ligado a otro concepto de moda, la calidad, ya que esta sólo es posible en la medida que se asegura el equilibrio, la uniformidad y la efectividad.

El modelo virtual, requiere de la estandarización, no solo para atender a las políticas económicas nacionales e internacionales de competitividad, sino porque es la única forma cómo funcionan las propuestas de educación masiva y apuestas centradas en las TIC. Por tanto, una educación preocupada en atender los aspectos de mercado y técnicos; datos, procesos, organización y tecnología, fácilmente abandona su esencia, lo académico-investigativo.

Privilegia evaluaciones de tipo cuantitativo: la educación masificada y estandarizada se centra en la transmisión de información o en los mejores casos en la transmisión de conocimiento. De ahí, las diversas evaluaciones cuantitativas implementadas por el sistema educativo, en las que lo único que se pone a prueba es la capacidad de memorizar o copiar, constituyendo un deprimente indicativo de las deficiencias de la educación que considera, que lo que no se recuerda no se sabe, en lugar de enseñar a 
razonar, a argumentar o mínimo a que los estudiantes entiendan lo que leen. (Ospina, 2012: 46-47)

Santos plantea una serie de patologías en la educación de todos los niveles con relación a los procesos evaluativos y que preocupan porque son indicativos de un problema serio de salud del sistema educativo. Llama la atención lo que implica la evaluación para las entidades educativas, para los docentes y para los estudiantes, indicando que con este ejercicio simplemente se replica la visión autoritaria, jerárquica, injusta y poco democrática cómo y con qué se evalúa (Santos, s.f: web).

En este sentido, la evaluación no es una medida que fortalezca la calidad del proceso educativo en las entidades de nivel superior; por el contrario, las calificaciones se ligan directamente a una política desigual que fomenta la existencia de profundas discrepancias entre la misión y el quehacer académico, sobre todo porque las evaluaciones no son objetivas, inocentes o ingenuas, ya que por su origen, los lineamientos provienen de instancias administrativas, y por tanto se le supedita a una multiplicidad de mecanismos y normas que provienen de esta área, que es quien maneja y controla la evaluación. En este marco, las evaluaciones vinculan rendimiento económico con los resultados académicos y no con los procesos, por lo que se desvirtúa el proceso de enseñanza aprendizaje.

Vigilancia institucional a estudiantes y docentes: reconocer que buena parte de la información privada que circula por las redes con nuestro beneplácito es de alguna manera, asumir sus consecuencias, ¿pero qué sucede cuando se publica información sin ser consultados? o ¿cuándo se vigilan nuestras actuaciones a través de los sistemas informáticos? Al parecer no hemos entendido sus implicaciones, sólo cuando ya es demasiado tarde. Las reglamentaciones frente al -acceso abusivo a un sistema informático- son aún muy débiles, tal vez el desarrollo vertiginoso de las TIC nos cogió de sorpresa o quizá no existe el interés de los diversos poderes en reglamentarlo. En la educación virtual, éste es un aspecto que se debería considerar seriamente.

Mediante la programación dirigida, los procesos de interacción grupal se reducen y restringen a modelos determinados hasta en las opciones más personales. Esta operación se hace de manera sutil, a través del efecto que ejerce el modelo. Los recursos o mediaciones en algunas universidades son definidos por los técnicos y administrativos no por los docentes, por tanto existe una vigilancia continúa hacia el educando, que parte de una educación en la cual se ejerce un control directo desde la institución y donde el aprendizaje ya no es dirigido solamente por el profesor.

La mecanización lleva a la rapidez y la eficiencia: no se puede desconocer que el uso de la tecnología trae consigo el quiebre del tiempo y el espacio, frente a lo cual se imponen nuevas formas de relacionamiento, de conocimiento y de desarrollo; siendo la rapidez, la eficiencia y la superación de las barreras geográficas, los mayores aliados para la productividad que necesitan las empresas, en detrimento de los procesos de pensamiento, de bienestar físico, de equilibrio eco-sistémico y de la construcción de tejido social.

El gran problema que enfrenta la universidad virtual es que el paradigma dominante no ha muerto, resurge con mayor ahínco, ya que se sustenta en modelos económicos posmodernistas y globalizados, convirtiéndose a la vez en el principal modelo peda- 
gógico, repitiendo y profundizando los mismos errores de la educación presencial, pues sus propuestas no atienden a la integralidad del ser humano, sino a lo instrumental y cognitivo. En últimas, se busca alta producción al implementar los principios de eficiencia, la evidencia observable y los resultados en el corto plazo. El interés está en tener gente que principalmente produzca, pero que muy poco piense, sea sensible, crítica y menos cuestione o vaya en contra del statu quo. (Moreno, et al, 2005: 74).

Mientras la educación continúe fomentando la competitividad, el éxito personal y la formación de sujetos para el mercado laboral, no será posible la construcción de tejido social, y "para ello no necesitamos técnicos ni operarios sino ciudadanos". (Ospina, 2012: 26)

Las mediaciones son definidas por los técnicos o administrativos: la educación del futuro enfila sus baterías hacia lo técnico-administrativo en detrimento de lo académico; normativiza y estandariza todos los actos pedagógicos, continúa fomentando la investigación cuantitativa, construye bases de datos para el control permanente de la información que circula por las redes, dispone y vigila las actividades académicas de los estudiantes y docentes, lo que a la larga se convierte en la justificación perfecta para la puesta en marcha de modelos pedagógicos homogeneizantes. Así, los académicos se transforman lentamente en operarios de la enseñanza y los estudiantes en clientes o consumidores.

La tecnología como fin y no como medio: la megauniversidad, la universidad virtual, la educación e-learning, la educación a distancia o cualquier otro nombre que plantee una educación centrada en el uso intensivo de las TIC, no deja de imponerse de manera avasalladora al estudiante y al docente, ahora convertidos en usuarios.

Desde la virtualidad, toman mayor relevancia los flujos y circuitos, ya que estos son los que permiten elaborar, editar, copiar y socializar los productos académicos, además facilitan la interconexión de las relaciones sociales, por lo que toda actividad está programada y es obligatoria. La programación hace desaparecer lo espontáneo, debido a que los elementos que están en la plataforma (foro, chat, video y demás) deben ser usados de los modos y en los tiempos establecidos de acuerdo con el diseño instruccional y son los medios a través de los cuales se logra la comunicación entre docente/estudiante, estudiante/estudiante y docente/docente, perdiéndose la espontaneidad y creatividad en el uso de los recursos educativos.

A medida que los flujos y circuitos se apropian del tejido social, se busca disimularlos más, la opacidad y el secreto provienen de la extrema sofisticación de la técnica. Se sabe poco de la programación, del funcionamiento de la plataforma virtual, del colapso del sistema de conexión, de la capacidad del servidor, de los bloqueos de información, de los virus informáticos, que dejan al estudiante-usuario, y en menor medida al docente-usuario, en una relación de dependencia pasiva, reducidos a la impotencia ante la complejidad de tales mecanismos técnicos y tecnológicos. Recordemos "Los cambios no provienen de las máquinas, los cambios son de los seres humanos que dan sentido a cualquier acto educativo". "Pedagogía antes que tecnología". (Prieto, 2006: 90)

De baja calidad: la ineficacia social del conocimiento universitario, evidenciado con mayor rigor en la educación virtual, es un elemento central que define la calidad de la educación superior porque lo convierte en un conocimiento aislado, válido so- 
lamente dentro de la misma institucionalidad, rompiendo sus relaciones con la sociedad. Con frecuencia se evade este aspecto central de la educación en todos los niveles, pero en la universidad virtual se hace más crítico, porque se han construido imaginarios que minan su calidad, al plantear que es una educación más económica, de menor esfuerzo y dirigida a quienes tienen poco tiempo para estudiar.

\section{Conclusiones}

Se requiere volver al sistema educativo y con mayor razón a la educación virtual, pues resulta evidente que la mediación tecnológica es un buen aliado (cuando se sabe utilizar) pero no es el fin último de la educación. Por tanto, se deben establecer políticas que incluyan el bienestar integral de los estudiantes, a través de la promoción de hábitos saludables, del afecto hacia los otros y hacia la naturaleza, la conservación de las tradiciones y la mesura. Lo fundamental está en reforzar un sistema de valores éticos y establecer criterios claros que conduzcan a despertar el sentido crítico de la sociedad.

No se puede seguir alimentando el imaginario que existe frente a esta modalidad educativa: la universidad virtual es más costosa, exigente y requiere mayor dedicación que la presencial porque se basa en procesos de autorregulación, autonomía y autodisciplina por parte del estudiante, con un docente que esté presente para apoyarlo en el proceso de aprendizaje, permitiendo que confronte con preguntas, incitándolo a buscar respuestas en sí mismo y en sus experiencias cotidianas.

Si las universidades se olvidan de los múltiples saberes, escenarios y sujetos, estarán condenadas a desaparecer o convertirse en una serie de tranquilos módulos de oficina por donde se pasearan las exigentes lógicas empresariales. Se trata de ver en la educación superior y específicamente en la universidad virtual, un espacio de producción de conocimiento y creatividad, que potencialice las inteligencias colectivas.

\section{Referencias bibliográficas}

BARBERO, Jesús (s.f): "Figuras del desencanto" en Revista Número: http://www.revistanumero.com/36fig.htm. [Fecha de consulta: 15 de abril de 2012]

DURÁN, José (2005): "Heramientas educativas en internet" en SEECI: http://www.ucm.es/info/seeci/Numeros/Numero 12/DATOS.htm. [Fecha de consulta: 25 de octubre de 2012]

GALINDO, Luis (1998): Técnicas de investigación. En sociedad, cultura y comunicación. México, Pearson.

LORA, Jorge y RÉCENDEZ, Cristina (2003): "La universidad neoliberal y la crisis educativa. De cómo año tras año llegaron las evaluaciones". Reflexión Política, $\mathrm{n}^{\circ}$ 10. Año 5. Colombia, IEP-UNAB, pp. 70-79

MORENO, Prudenciano y SOTO, Graciela (2005): "Una mirada reflexiva y crítica al enfoque por competencias" en Redes: http://www.redes-cepalcala.org/.../COMPETENCIAS/.. [Fecha de consulta: 17 de septiembre de 2011]

OSPINA, William (2012): La lámpara maravillosa. Bogotá, Mondadori. 
PISCITELLI, Alejandro (2011): "Desafíos educativos en la era tecnológica" en RELPE: http://www.educarchile.cl/Portal.Base/Web/VerContenido.aspx?ID=209119. [Fecha de consulta: 10 de agosto de 2012]

PRIETO, Daniel y VAN DE POL, Peter (2006): E-Learning comunicación y educación el diálogo continúa en el ciberespacio. Costa Rica, RNTC.

SANTOS, Miguel (s.f): "Patología general de la evaluación educativa" en Dialnet: http://www.dialnet.unirioja.es/descarga/articulo/48299.pdf. [Fecha de consulta: 19 de febrero de 2011]

TAMAYO, Mario (2003): El proceso de la investigación científica. México, Limusa.

VAQUERIZO, María Belén (2012): "Enseñanza-aprendizaje con web 2.0 y 3.0" en Vivat Academia: http://www.ucm.es/info/vivataca/numeros/n117E/DATOSS.htm. [Fecha de consulta: 27 de octubre de 2012]

\section{Nelly TOVAR TORRES}

Universidad Nacional Abierta y a Distancia - UNAD, Colombia

Magister en Investigación Social Interdisciplinaria y Especialista en Multimedia Educativa. Pregrados en Comunicación Social-Periodismo y Promoción Social. Amplia experiencia en diseño, gestión/evaluación de proyectos sociales y educativos, como docente universitaria e investigadora.

nelly.tovar@unad.edu.co 